\title{
A PIECEWISE HyPERBoliC MAP WITH ABSOLUTELY CONTINUOUS INVARIANT MEASURE
}

\author{
PERSSON, TOMAS
}

Preprints in Mathematical Sciences

2005:29

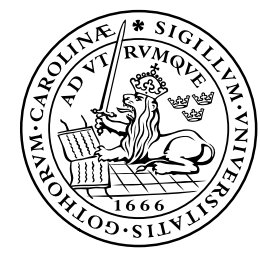

LUND INSTITUTE OF TECHNOLOGY Lund University

Centre for Mathematical Sciences

Mathematics 



\title{
A piecewise hyperbolic map with absolutely continuous invariant measure
}

\author{
Tomas Persson \\ Centre for Mathematical Sciences, Department of Mathematics, \\ Lund Institute of Technology, P.O. Box 118, SE-22100 Lund, Sweden \\ tomasp@maths.lth.se
}

October 24, 2005

\begin{abstract}
We consider a class of non invertible piecewise affine hyperbolic endomorphisms with singularities and show that for an open set of parameters there exists almost surely an absolutely continuous invariant measure. Also, exponential decay of correlations is proved for Hölder continuous functions.
\end{abstract}

Acknowledgement. Thank you Jörg Schmeling for great help.

\section{Introduction}

In [1], Alexander and Yorke considered a one parameter class of maps called the fat baker's transformations. These maps are piecewise affine maps of the square with one expanding and one contracting direction. Their results together with the result of Solomyak in [14], imply that for an open set of parameters, almost surely there is an absolutely continuous invariant measure. The fat baker's transformations are a special case of the Belykh map, introduced in [5] by Belykh. Schmeling and Troubetzkoy considered in [13] the Belykh map for a wider range of parameters. It was further investigated in [12].

In this article we consider a three parameter class of endomorphisms similar to the Belykh map. In fact, this class has a non empty intersection with the class of Belykh maps considered in [13] and [12] and it contains the fat baker's transformations. We show that there is an open set of parameters for which there is almost surely an absolutely continuous invariant measure.

Similar results in two dimensions, but in the case of expanding maps, were independently obtained by Buzzi in [3] and Tsujii in [15]. The corresponding results for arbitrary dimension are in [2] and [16].

In [17] Young introduced a method using a tower construction for proving exponential decay of correlations for a wide range of hyperbolic maps. Among other examples in the article she uses the method to prove exponential decay of correlations for a class of piecewise $C^{2}$ maps in two dimensions. Buzzi and Keller proved in [4] that a class of piecewise affine and expanding maps have exponential decay of correlations. In Section 5 the method of Young is adopted to show exponential decay of correlations for our class of maps. 


\section{The class of endomorphisms}

Put $Q=[-1,1]^{2}$ and $S=([-1,0] \times\{-\kappa\}) \cup(\{0\} \times[-|\kappa|,|\kappa|]) \cup([0,1] \times\{\kappa\})$. Let $Q_{1}$ and $Q_{-1}$ be the upper respectively the lower connected component of the set $Q \backslash S$.

Consider the class of maps $f: Q \backslash S \rightarrow Q$ defined by

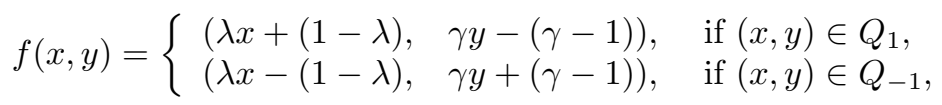

where the parameters are $\frac{1}{2}<\lambda \leq 1,-1<\kappa<1$ and $1<\gamma \leq \frac{2}{1+|\kappa|}$. See Figure 1.

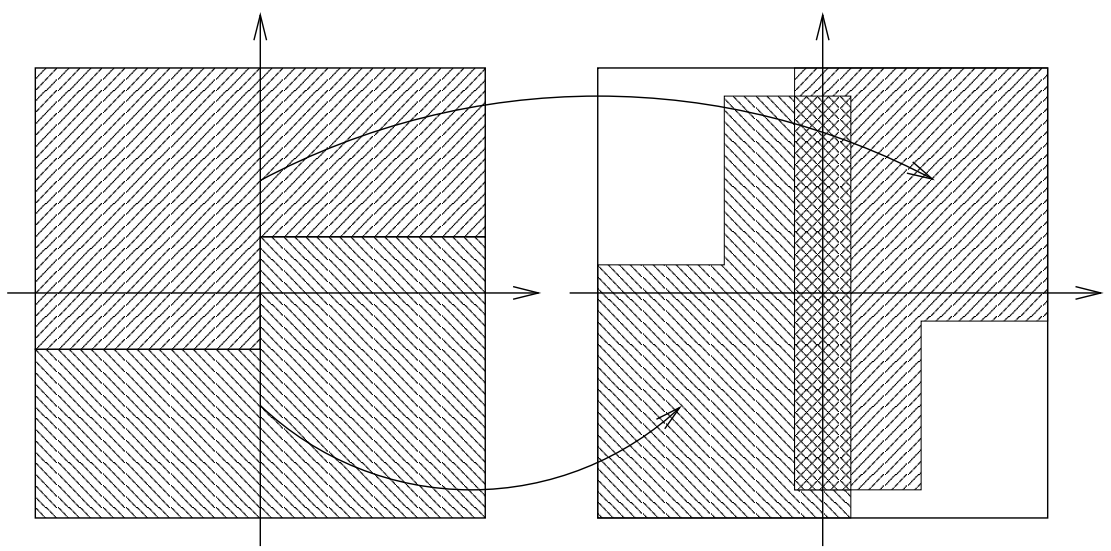

Figure 1: The map $f$ for $\gamma=\frac{3}{2}, \lambda=\frac{9}{16}$ and $\kappa=\frac{1}{4}$.

The set $S$ is the discontinuity set. Let $S_{\mathrm{v}}=\{0\} \times[-|\kappa|,|\kappa|]$ be its vertical component and $S_{\mathrm{h}}=([-1,0] \times\{-\kappa\}) \cup([0,1] \times\{\kappa\})$ be its horizontal component.

With a little more work the method of this paper can be used for maps with singularity sets with more than one jump. The only difficulty is to show that the complete backward orbit of the vertical components of the singularity consists of finitely many pieces.

The main difficulty in working with the map $f$ is that it is not invertible. To handle this problem we lift the map $f$ to an invertible map $\hat{f}$ on the three dimensional cube. The idea is to prove the desired result for $\hat{f}$ and then project the result on $f$. This idea has previously been used in [13] and [12].

The map $\hat{f}$ is defined as follows. Put $\hat{Q}=[-1,1]^{3}$ and $\hat{Q}_{i}=Q_{i} \times[-1,1]$, $i=-1,1$. Define $\hat{f}: \hat{Q}_{1} \cup \hat{Q}_{-1} \rightarrow \hat{Q}$ by

$$
\hat{f}\left(x_{1}, x_{2}, x_{3}\right)= \begin{cases}\left(f\left(x_{1}, x_{2}\right), \tau x_{3}+(1-\tau)\right), & \text { if }\left(x_{1}, x_{2}, x_{3}\right) \in \hat{Q}_{1}, \\ \left(f\left(x_{1}, x_{2}\right), \tau x_{3}-(1-\tau)\right), & \text { if }\left(x_{1}, x_{2}, x_{3}\right) \in \hat{Q}_{-1},\end{cases}
$$

where $\tau$ is chosen so small that $\hat{f}$ is invertible on its image, that is $\tau<\frac{1}{2}$. See Figure 2. Denote by $\pi$ the projection from $\hat{Q}$ to $Q$, defined by $\pi\left(x_{1}, x_{2}, x_{3}\right)=$ $\left(x_{1}, x_{2}\right)$. 


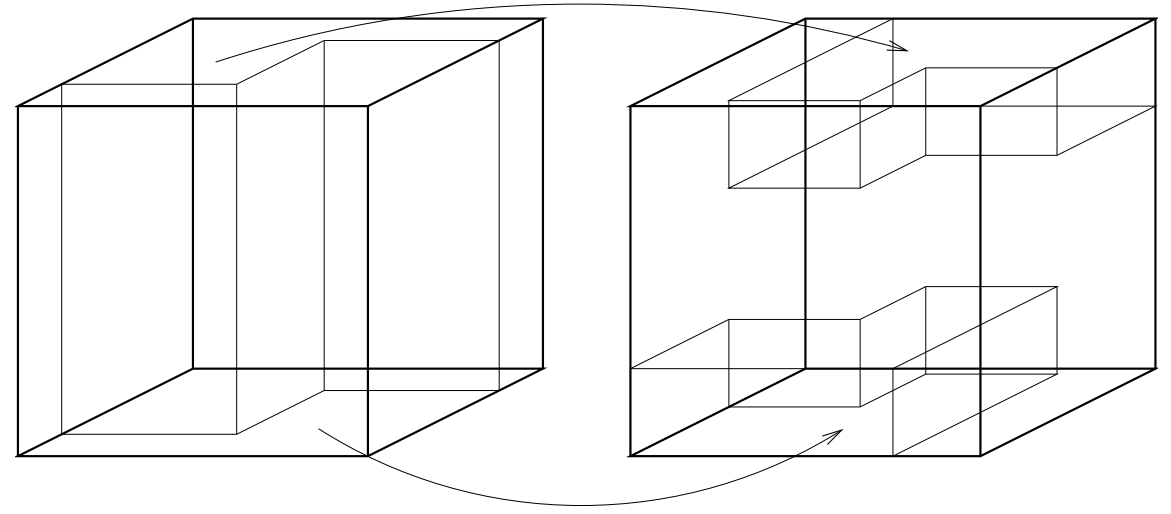

Figure 2: The map $\hat{f}$ for $\gamma=\frac{3}{2}, \lambda=\frac{3}{4}, \kappa=\frac{1}{4}$ and $\tau=\frac{1}{4}$.

Given a sequence $\underline{i} \in \Sigma_{2}=\{-1,1\}^{\mathbb{N}}$, define the cylinder set

$$
\hat{R}_{\underline{i}}^{l, m}(\lambda, \gamma, \kappa)=\left\{\hat{x} \in \hat{Q} \mid \hat{f}_{\lambda, \gamma, \kappa}^{n}(\hat{x}) \in \hat{Q}_{i_{n}}, n=-m,-m+1, \ldots,-l\right\},
$$

where $0 \leq l<m \in \mathbb{N}$. We will write $\hat{R}_{\underline{i}}^{m}(\lambda, \gamma, \kappa)$ for $\hat{R}_{\underline{i}}^{0, m}(\lambda, \gamma, \kappa)$. Let

$$
\Sigma_{\lambda, \gamma, \kappa}=\left\{\underline{i} \in \Sigma_{2} \mid R_{\underline{i}}^{l, m}(\lambda, \gamma, \kappa) \neq \emptyset \forall l, m \in \mathbb{Z}\right\}
$$

be the coding of the system $\hat{f}_{\lambda, \gamma, \kappa}$. Denote by $\sigma$ the left shift.

The set

$$
\hat{\Lambda}_{\lambda, \gamma, \kappa}=\bigcap_{n \in \mathbb{Z}} \hat{f}_{\lambda, \gamma, \kappa}^{n}(\hat{Q})
$$

is the attractor of $\hat{f}_{\lambda, \gamma, \kappa}$. It is easy to see that the map $\rho_{\lambda, \gamma, \kappa}: \Sigma_{\lambda, \gamma, \kappa} \rightarrow \hat{Q}$ defined by $\rho_{\lambda, \gamma, \kappa}: \underline{i} \mapsto\left(x_{1}, x_{2}, x_{3}\right)$ where

$$
\begin{aligned}
& x_{1}=\frac{1-\lambda}{\lambda} \sum_{n=1}^{\infty} i_{-n} \lambda^{n}, \\
& x_{2}=\frac{\gamma-1}{\gamma} \sum_{n=0}^{\infty} i_{n} \gamma^{n}, \\
& x_{3}=\frac{1-\tau}{\tau} \sum_{n=1}^{\infty} i_{-n} \tau^{n},
\end{aligned}
$$

satisfies $\rho_{\lambda, \gamma, \kappa}(\sigma(\underline{i}))=\hat{f}_{\lambda, \gamma, \kappa}\left(\rho_{\lambda, \gamma, \kappa}(\underline{i})\right)$ for all $\underline{i} \in \Sigma_{\lambda, \gamma, \kappa}$ and $\rho_{\lambda, \gamma, \kappa}\left(\Sigma_{\lambda, \gamma, \kappa}\right)=$ $\hat{\Lambda}_{\lambda, \gamma, \kappa}$. Hence $\hat{\Lambda}_{\lambda, \gamma, \kappa}$ is the set

$$
\begin{aligned}
& \hat{\Lambda}_{\lambda, \gamma, k}=\left\{\left(x_{1}, x_{2}, x_{3}\right) \mid \exists \underline{i} \in \Sigma_{\lambda, \gamma, \kappa}: x_{1}=\frac{1-\lambda}{\lambda} \sum_{n=1}^{\infty} i_{-n} \lambda^{n}\right. \\
&\left.x_{2}=\frac{\gamma-1}{\gamma} \sum_{n=0}^{\infty} i_{n} \gamma^{n}, x_{3}=\frac{1-\tau}{\tau} \sum_{n=1}^{\infty} i_{-n} \tau^{n}\right\} .
\end{aligned}
$$


Let $\hat{\nu}$ denote the normalised Lebesgue measure on $\hat{Q}$ and for $n \in \mathbb{N}$ define $\hat{\nu}_{n}=\hat{\nu} \circ \hat{f}^{-n}$. The sequence of measures

$$
\hat{\mu}_{m}=\frac{1}{m} \sum_{n=0}^{m-1} \hat{\nu}_{n}
$$

converges weakly to the SBR-measure, $\hat{\mu}_{\mathrm{SBR}}$. In [13] it is shown that the measure $\mu_{\mathrm{SBR}}=\hat{\mu}_{\mathrm{SBR}} \circ \pi^{-1}$ is the SBR-measure of $f$. It has the property that the conditional measures on the unstable manifolds are absolutely continuous with respect to Lebesgue measure.

The map $f$ satisfies the conditions (H1), (H5)-(H9) in [9] and this implies that there exists $C, q>0$ such that $\nu\left(f^{-n} U(S, \varepsilon)\right) \leq C \varepsilon^{q}$ for all $n>0$, where $U(S, \varepsilon)$ denotes the $\varepsilon$-neighborhood of $S$. This implies that $\mu_{\mathrm{SBR}}(\Lambda)=$ $\hat{\mu}_{\mathrm{SBR}}(\hat{\Lambda})=1$. In $[9]$ it is also shown that $h_{\hat{\mu}_{\mathrm{SBR}}}=h_{\mu_{\mathrm{SBR}}}=\log \gamma$.

Let $x \in Q$. We define the local stable manifold $W^{\mathrm{s}}(x)$ of $x$ to be the largest connected subset of $\left\{y \in Q \mid d\left(f^{n}(x), f^{n}(y)\right) \rightarrow 0, n \rightarrow \infty\right\}$ containing $x$. If the set $\left\{y \in W^{\mathrm{s}}(x) \mid d(x, y)<\delta\right\}$ is a connected curve of length $2 \delta$ then define $W_{\delta}^{\mathrm{s}}(x)=\left\{y \in W^{\mathrm{s}}(x) \mid d(x, y)<\delta\right\}$ to be the stable manifold of $x$ with length $2 \delta$. Otherwise we say that $W_{\delta}^{\text {s }}(x)$ does not exist.

Since $f$ is not invertible we can not define the local unstable manifold of $x$ in the usual way. Let

$$
D_{\delta}^{-}=\left\{x \mid d\left(f^{-n}(x), S\right)>\delta \gamma^{-n} \forall n \geq 0\right\} .
$$

This set has positive Lebesgue measure if $\delta$ is taken sufficiently small, see [9]. It is even true that

$$
\mu_{\mathrm{SBR}}\left(\bigcup_{l=1}^{\infty} D_{l^{-1}}^{-}\right)=1
$$

If $x=\left(x_{1}, x_{2}\right) \in D_{\delta}^{-}$then for any $y=\left(y_{1}, y_{2}\right)$ with $y_{1}=x_{1}$ and $\left|x_{2}-y_{2}\right|<\delta$ we have $d\left(f^{-n}(x), f^{-n}(y)\right)<\delta \gamma^{-n}$ for all $n \geq 0$. We can thus define the unstable manifold of $x=\left(x_{1}, x_{2}\right) \in D_{\delta}^{-}$of length $\delta$ to be the set

$$
W_{\delta}^{\mathrm{u}}(x)=\left\{y=\left(y_{1}, y_{2}\right)\left|y_{1}=x_{1},\right| x_{2}-y_{2} \mid<\delta\right\} .
$$

If $x \notin D_{\delta}^{-}$then we say that $W_{\delta}^{\mathrm{u}}(x)$ does not exist.

\section{Absolutely continuous invariant measure}

We will prove the following theorem.

Theorem 1. There is an open ball $P \subset\{\lambda, \gamma, \kappa\}$ such that $\mu_{\mathrm{SBR}} \ll \nu$ for a.e. $(\lambda, \gamma, \kappa) \in P$.

There are parameters in the set $\{\gamma \lambda>1, \kappa=0\}$ for which the SBR-measure is not absolutely continuous. In [1], Alexander and Yorke point out that if $\kappa=0$, $\gamma=2$ and $\lambda^{-1}$ is a Pisot number, then the SBR-measure is singular with respect to Lebesgue measure. If $\gamma \lambda<1$ then there can not be any absolutely continuous invariant measure $\mu$, since then the Lebesgue measure of $f^{n}(Q)$ converges to zero but $\mu\left(f^{n}(Q)\right)=1$ by invariance. 


\section{Proof of the theorem}

To prove Theorem 1 the method of Peres and Solomyak in [8] will be used. This is a simplified version of the method used by Solomyak in [14]. Peres and Solomyak showed the almost surely absolutely continuity of a Bernoulli convolution when the associated shift space is the full two-shift $\Sigma_{2}$. In this proof we have to work with the more restricted shift space $\Sigma_{\lambda, \gamma, \kappa}$ and this introduces more difficulties in the proof. Also, it is necessary to controll how the shift space $\Sigma_{\lambda, \gamma, \kappa}$ depends on the parameters.

The following Lemma from [14] will be used in the proof of Theorem 1.

Lemma 1. Let $\varepsilon>0$ be fixed and $I \subset\left(\gamma^{-1}+\varepsilon, 0.64\right)$. There is a constant $c$ such that for any $k \in \mathbb{N}$ and any $\left\{a_{i}\right\}_{i=0}^{\infty} \in\{-1,0,1\}^{\mathbb{N}}$ the following estimate is valid.

$$
\int_{I} \chi_{\left\{\lambda|| \lambda^{k}+\sum_{i=k+1}^{\infty} a_{i} \lambda^{i} \mid<r\right\}} \leq c\left(\gamma^{-1}+\varepsilon\right)^{-k} r .
$$

Let $\gamma, \kappa$ be fixed and $I \subseteq(0.5,0.64)$ any interval such that $\{\gamma\} \times I \times\{\kappa\} \subset P$, where the set $P$ will be chosen later.

Let $B_{r}(t)=[t-r, t+r]$ and let

$$
D\left(\mu_{\mathrm{SBR}}^{\lambda, \mathrm{s}, x}, t\right)=\liminf _{r \rightarrow 0} \frac{\mu_{\mathrm{SBR}}^{\lambda, \mathrm{s}, x}\left(B_{r}(t)\right)}{2 r}
$$

denote the lower derivative of the measure $\mu_{\mathrm{SBR}}^{\lambda, \mathrm{s}, x}$ - the conditional measure of $\mu_{\mathrm{SBR}}^{\lambda}$ with respect to the local stable manifold $W^{\mathrm{s}}(x)$. The partition of $Q$ into local stable manifolds is clearly measurable so the conditional measures exist and we can use them as follows. We want to prove that for a.e. $\lambda$ there is a set $\Omega_{\lambda}$ such that $\mu_{\mathrm{SBR}}^{\lambda}\left(\Omega_{\lambda}\right)>0$ and

$$
\int_{\Omega_{\lambda}} D\left(\left.\mu_{\mathrm{SBR}}^{\lambda, \mathrm{s}, x}\right|_{\Omega_{\lambda}}, y\right) \mathrm{d} \mu_{\mathrm{SBR}}^{\lambda, \mathrm{s}, x}(y)<\infty
$$

holds for a.e. $x$. This implies that the measure $\mu_{\mathrm{SBR}}^{\lambda, \mathrm{s}, x}$ restricted to the set $\Omega_{\lambda}$ is absolutely continuous for a.e. $x$. Since the conditional measures on the unstable manifolds are absolutely continuous with respect to Lebesgue measure, this implies that $\left.\mu_{\mathrm{SBR}}^{\lambda}\right|_{\Omega_{\lambda}}$ is absolutely continuous with respect to Lebesgue measure. Since $\mu_{\mathrm{SBR}}^{\lambda}\left(\Omega_{\lambda}\right)>0$, ergodicity then implies that this also holds for the measure $\mu_{\mathrm{SBR}}^{\lambda}$.

Fatou's lemma implies that in order to prove (1) it suffices to prove that

$$
\liminf _{r \rightarrow o} \frac{1}{r} \int_{\Omega_{\lambda}} \mu_{\mathrm{SBR}}^{\lambda, \mathrm{s}, x}\left(\Omega_{\lambda} \cap B_{r}(y)\right) \mathrm{d} \mu_{\mathrm{SBR}}^{\lambda, \mathrm{s}, x}(y)<\infty .
$$

We may rewrite this as

$$
\liminf _{r \rightarrow 0} \frac{1}{r} \int_{\Omega_{\lambda}} \int_{\Omega_{\lambda}} \chi_{\left\{\left|y_{1}-z_{1}\right|<r\right\}} \mathrm{d} \mu_{\mathrm{SBR}}^{\lambda, \mathrm{s}, x}(z) \mathrm{d} \mu_{\mathrm{SBR}}^{\lambda, \mathrm{s}, x}(y)<\infty .
$$

We will choose a class of functions $x: I \rightarrow Q$ such that $x(\lambda) \in \Omega_{\lambda}$ and prove that the following estimate is valid

$$
\liminf _{r \rightarrow 0} \frac{1}{r} \int_{I} \int_{\Omega_{\lambda}} \int_{\Omega_{\lambda}} \chi_{\left\{\left|y_{1}-z_{1}\right|<r\right\}} \mathrm{d} \mu_{\mathrm{SBR}}^{\lambda, \mathrm{s}, x(\lambda)}(z) \mathrm{d} \mu_{\mathrm{SBR}}^{\lambda, \mathrm{s}, x(\lambda)}(y) \mathrm{d} \lambda<\infty .
$$




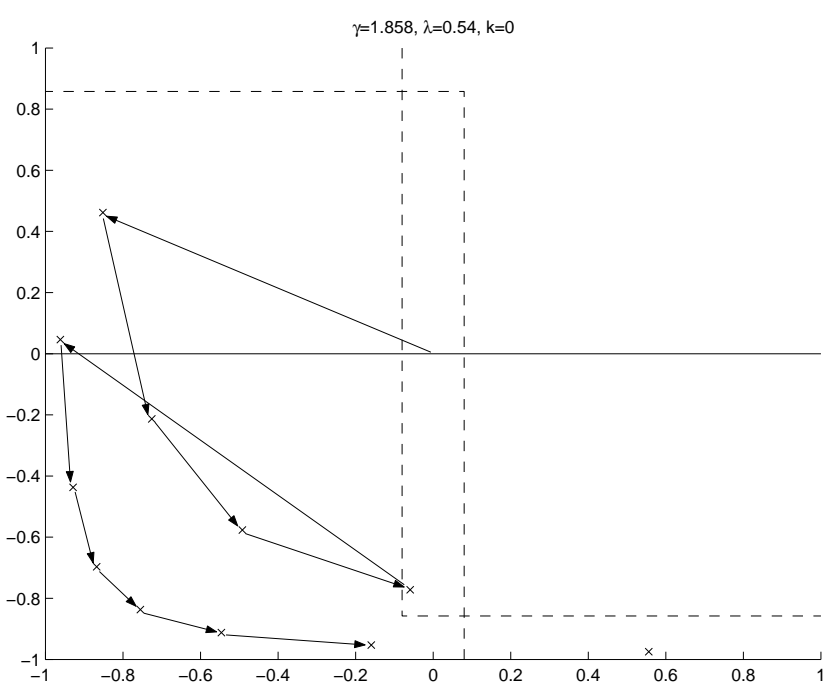

Figure 3: Some inverse images of $S_{\mathrm{v}}$.

This then implies that $\mu_{\mathrm{SBR}} \ll \nu$ for a.e. $\lambda \in I$. Instead of proving (3) we use that $\mu_{\mathrm{SBR}}=\hat{\mu}_{\mathrm{SBR}} \circ \pi^{-1}$ and prove the equivalent condition

$$
\liminf _{r \rightarrow 0} \frac{1}{r} \int_{I} \int_{\hat{\Omega}_{\lambda}} \int_{\hat{\Omega}_{\lambda}} \chi_{\left\{\left|y_{1}-z_{1}\right|<r\right\}} \mathrm{d} \hat{\mu}_{\mathrm{SBR}}^{\lambda, \mathrm{s}, \hat{x}(\lambda)}(\hat{z}) \mathrm{d} \hat{\mu}_{\mathrm{SBR}}^{\lambda, \mathrm{s}, \hat{x}(\lambda)}(\hat{y}) \mathrm{d} \lambda<\infty
$$

To prove (4) the symbolic coding $\Sigma_{\lambda, \gamma, \kappa}$ will be used. Since we have $\gamma$ and $\kappa$ fixed and vary $\lambda$ it is the dynamics taking place in the horizontal direction that is crucial. It is hence the dynamical behaviour of the vertical component of the singularity $S_{\mathrm{v}}$ that is important. Below, we will choose a set $P$ in which $S_{\mathrm{v}}$ behaves as if it does not exist.

One checks with a numerical calculation that for $\gamma=1.858, \lambda=0.54$ and $\kappa=0$ there are only finitely many points in the complete backward orbit of the vertical piece of the singularity, $S_{\mathrm{v}}$. Numerics are only used to see this in an easy way. It does not influence on the rigorousity of the proof. In this case, when $\kappa=0$, we have $S_{\mathrm{v}}=\{0\}$. Figure 3 and 4 illustrate this by showing the two possible paths of $\{0\}$ starting in $Q_{1}$. The points in the backward orbit of $S_{\mathrm{v}}$ are marked with $\times$ in the figures. The dashed lines are the border of the sets $f\left(Q_{1}\right)$ and $f\left(Q_{-1}\right)$. The arrows shows how the points are mapped by $f^{-1}$. Each path drawn in the figures terminates after finitely many steps, after which there are no more inverse images.

The numerical evaluations of the points are in Table 1 and Table 2. Comparing these values with

$$
\begin{aligned}
& f\left(Q_{1}\right)=[1-2 \lambda, 1] \times[1-\gamma, 1]=[-0.08,1] \times[-0.858,1], \\
& f\left(Q_{-1}\right)=[-1,2 \lambda-1] \times[-1, \gamma-1]=[-1,0.08] \times[-1,0.858],
\end{aligned}
$$

shows that there are no more points in the backward orbit of $S_{\mathrm{v}}$ and that these points are bounded away from the set $f(S)$. This allow us to draw the following 


\begin{tabular}{|c|c|c|c|}
\hline iterate & coordinates & $\begin{array}{l}\text { numerical } \\
\text { values }\end{array}$ & contained in \\
\hline 0 & $\begin{array}{l}0 \\
0\end{array}$ & $\begin{array}{l}\overline{0} \\
0\end{array}$ & $f\left(Q_{1}\right) \cap f\left(Q_{-1}\right)$ \\
\hline-1 & $\frac{\frac{-1+\lambda}{\lambda}}{\frac{-1+\gamma}{\gamma}}$ & $\begin{array}{r}-0.8519 \\
0.4618\end{array}$ & $f\left(Q_{-1}\right) \backslash f\left(Q_{1}\right)$ \\
\hline-2 & $\begin{array}{l}\frac{-1+2 \lambda-\lambda^{2}}{\lambda^{2}} \\
\frac{-1+2 \gamma-\gamma^{2}}{2}\end{array}$ & $\begin{array}{l}-0.7257 \\
-0.2132\end{array}$ & $f\left(Q_{-1}\right) \backslash f\left(Q_{1}\right)$ \\
\hline-3 & $\begin{array}{l}\frac{-1+2 \lambda-\lambda^{3}}{\lambda^{3}} \\
\frac{-1+2 \gamma-\gamma^{3}}{\gamma^{3}}\end{array}$ & $\begin{array}{l}-0.4919 \\
-0.5766\end{array}$ & $f\left(Q_{-1}\right) \backslash f\left(Q_{1}\right)$ \\
\hline-4 & $\begin{array}{l}\frac{-1+2 \lambda-\lambda^{4}}{\lambda^{4}} \\
\frac{-1+2 \gamma-\gamma^{4}}{\gamma^{4}}\end{array}$ & $\begin{array}{l}-0.05916 \\
-0.7721\end{array}$ & $f\left(Q_{1}\right) \cap f\left(Q_{-1}\right)$ \\
\hline-5 & $\begin{array}{l}\frac{-1+2 \lambda-2 \lambda^{4}+\lambda^{5}}{\lambda^{5}} \\
\frac{-1+2 \gamma-2 \gamma^{4}+\gamma^{5}}{\gamma^{5}}\end{array}$ & $\begin{array}{c}-0.9614 \\
0.04623\end{array}$ & $f\left(Q_{-1}\right) \backslash f\left(Q_{1}\right)$ \\
\hline-6 & $\begin{array}{l}\frac{-1+2 \lambda-2 \lambda^{4}+2 \lambda^{5}-\lambda^{6}}{\lambda^{6}} \\
\frac{-1+2 \gamma-\gamma^{4}+2 \gamma^{5}-\gamma^{6}}{\gamma^{6}}\end{array}$ & $\begin{array}{l}-0.9285 \\
-0.4369\end{array}$ & $f\left(Q_{-1}\right) \backslash f\left(Q_{1}\right)$ \\
\hline-7 & $\begin{array}{c}\frac{-1+2 \lambda-2 \lambda^{4}+2 \lambda^{5}-\lambda^{7}}{\lambda^{7}} \\
\frac{-1+2 \gamma-\gamma^{4}+2 \gamma^{5}-\gamma^{7}}{\gamma^{7}}\end{array}$ & $\begin{array}{l}-0.8677 \\
-0.6969\end{array}$ & $f\left(Q_{-1}\right) \backslash f\left(Q_{1}\right)$ \\
\hline-8 & $\begin{array}{c}\frac{-1+2 \lambda-2 \lambda^{4}+2 \lambda^{5}-\lambda^{8}}{\lambda^{8}} \\
\frac{-1+2 \gamma-\gamma^{4}+2 \gamma^{5}-\gamma^{8}}{\gamma^{8}}\end{array}$ & $\begin{array}{l}-0.7549 \\
-0.8369\end{array}$ & $f\left(Q_{-1}\right) \backslash f\left(Q_{1}\right)$ \\
\hline-9 & $\begin{array}{c}\frac{-1+2 \lambda-2 \lambda^{4}+2 \lambda^{5}-\lambda^{9}}{\lambda^{9}} \\
\frac{-1+2 \gamma-\gamma^{4}+2 \gamma^{5}-\gamma^{9}}{\gamma^{9}}\end{array}$ & $\begin{array}{l}-0.5462 \\
-0.9122\end{array}$ & $f\left(Q_{-1}\right) \backslash f\left(Q_{1}\right)$ \\
\hline-10 & $\begin{array}{c}\frac{-1+2 \lambda-2 \lambda^{4}+2 \lambda^{5}-\lambda^{10}}{\lambda^{10}} \\
\frac{-1+2 \gamma-\gamma^{4}+2 \gamma^{5}-\gamma^{10}}{\gamma^{10}}\end{array}$ & $\begin{array}{l}-0.1596 \\
-0.9528\end{array}$ & $f\left(Q_{-1}\right) \backslash f\left(Q_{1}\right)$ \\
\hline-11 & $\begin{array}{c}\frac{-1+2 \lambda-2 \lambda^{4}+2 \lambda^{5}-\lambda^{11}}{\lambda^{11}} \\
\frac{-1+2 \gamma-\gamma^{4}+2 \gamma^{5}-\gamma^{11}}{\gamma^{11}}\end{array}$ & $\begin{array}{r}0.5563 \\
-0.9746\end{array}$ & $Q \backslash\left(f\left(Q_{1}\right) \cup f\left(Q_{-1}\right)\right)$ \\
\hline
\end{tabular}

Table 1: The points in Figure 3. 


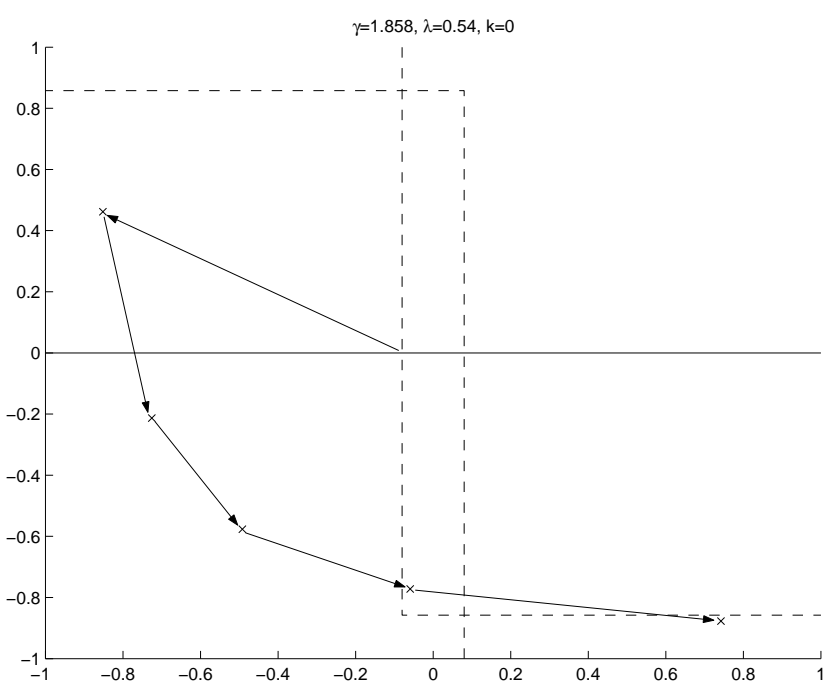

Figure 4: Yet some inverse images of $S_{\mathrm{v}}$.

\begin{tabular}{|c|c|c|c|}
\hline iterate & coordinates & $\begin{array}{l}\text { numerical } \\
\text { values }\end{array}$ & contained in \\
\hline 0 & $\begin{array}{l}0 \\
0\end{array}$ & $\begin{array}{l}0 \\
0\end{array}$ & $f\left(Q_{1}\right) \cap f\left(Q_{-1}\right)$ \\
\hline-1 & $\begin{array}{l}\frac{-1+\lambda}{\lambda} \\
\frac{-1+\gamma}{\gamma}\end{array}$ & $\begin{array}{r}-0.8519 \\
0.4618\end{array}$ & $f\left(Q_{-1}\right) \backslash f\left(Q_{1}\right)$ \\
\hline-2 & $\begin{array}{l}\frac{-1+2 \lambda-\lambda^{2}}{\lambda^{2}} \\
\frac{-1+2 \gamma-\gamma^{2}}{\gamma^{2}}\end{array}$ & $\begin{array}{l}-0.7257 \\
-0.2132\end{array}$ & $f\left(Q_{-1}\right) \backslash f\left(Q_{1}\right)$ \\
\hline-3 & $\begin{array}{l}\frac{-1+2 \lambda-\lambda^{3}}{\lambda^{3}} \\
\frac{-1+2 \gamma-\gamma^{3}}{\gamma^{3}}\end{array}$ & $\begin{array}{l}-0.4919 \\
-0.5766\end{array}$ & $f\left(Q_{-1}\right) \backslash f\left(Q_{1}\right)$ \\
\hline-4 & $\begin{array}{c}\frac{-1+2 \lambda-\lambda^{4}}{\lambda^{4}} \\
\frac{-1+2 \gamma-\gamma^{4}}{\gamma^{4}}\end{array}$ & $\begin{array}{l}-0.05916 \\
-0.7721\end{array}$ & $f\left(Q_{1}\right) \cap f\left(Q_{-1}\right)$ \\
\hline-5 & $\begin{array}{l}\frac{-1+2 \lambda-\lambda^{5}}{\lambda^{5}} \\
\frac{-1+2 \gamma-\gamma^{5}}{\gamma^{5}}\end{array}$ & $\begin{array}{r}0.7423 \\
-0.8773\end{array}$ & $Q \backslash\left(f\left(Q_{1}\right) \cup f\left(Q_{-1}\right)\right)$ \\
\hline
\end{tabular}

Table 2: The points in Figure 4. 
conclusion. There is an open neighborhood $U$ of $S_{\mathrm{v}}$ such that $f^{-N}(U)=\emptyset$ for $N$ sufficiently large.

By the continuous dependence on the parameters of the finitely many points in the backward orbit of $S_{\mathrm{v}}$, there exists an open ball $P \subset\{\gamma, \lambda, \kappa\}$ around $(\gamma, \lambda, \kappa)=(1.858,0.54,0)$ such that the backward orbit of $S_{\mathrm{v}}$ behaves in the same way for any $(\gamma, \lambda, \kappa) \in P$ in the following sense. For any $(\gamma, \lambda, \kappa) \in P$ the backward orbit of $S_{\mathrm{v}}$ contains finitely many pieces, each bounded away from $f(S)$, and there are uniform numbers $\alpha>0$ and $N>0$ such that $U=$ $([-\alpha, \alpha] \times[-|\kappa|,|\kappa|])$ satisfies $f^{-N}(U)=\emptyset$. This implies that $U \cap \Lambda_{\gamma, \lambda, \kappa}=\emptyset$ for any $(\gamma, \lambda, \kappa) \in P$.

Recall that we have the parameters $\gamma$ and $\kappa$ fixed and $I$ is an interval such that $\{\gamma\} \times I \times\{\kappa\} \subset P$. Partition $I$ into sub intervals $\left\{I_{t}\right\}_{t=1}^{p}$, such that $\left|I_{t}\right|<\frac{1}{15} \alpha$. This can be done so that $p<\frac{15|I|}{\alpha}+1$. For each $t=1, \ldots, p$ fix a $\lambda_{t} \in I_{t}$. The following lemma will provide sufficient control when changing the parameter $\lambda$.

Lemma 2. For any $t$ and any $\lambda, \lambda^{\prime} \in I_{t}$ the symbolic spaces $\Sigma_{\lambda, \gamma, \kappa}$ and $\Sigma_{\lambda^{\prime}, \gamma, \kappa}$ coincide.

Proof. A point $\hat{x}=\left(x_{1}, x_{2}, x_{3}\right) \in \hat{Q}$ lies in $\hat{\Lambda}_{\lambda_{t}}$ if and only if there is a sequence $\left\{i_{n}\right\}_{n \in \mathbb{Z}}$ such that

$$
x_{1}=\frac{1-\lambda_{t}}{\lambda_{t}} \sum_{n=1}^{\infty} i_{-n} \lambda_{t}^{n}, \quad x_{2}=\frac{\gamma-1}{\gamma} \sum_{n=0}^{\infty} i_{n} \gamma^{-n}, \quad x_{3}=\frac{1-\tau}{\tau} \sum_{n=1}^{\infty} i_{-n} \tau^{n}
$$

and $\hat{f}_{\lambda_{t}}^{n}(\hat{x}) \in \hat{Q}_{i_{n}}$ for all $n \in \mathbb{Z}$. Let $\hat{x}=\left(x_{1}, x_{2}, x_{3}\right) \in \hat{\Lambda}_{\lambda_{t}}$. We show that for any $\lambda^{\prime} \in I_{t}$ there is a point $\hat{x}^{\prime}=\left(x_{1}^{\prime}, x_{2}^{\prime}, x_{3}^{\prime}\right) \in \hat{\Lambda}_{\lambda^{\prime}}$ such that the corresponding sequence $\left\{i_{n}^{\prime}\right\}_{n \in \mathbb{Z}}$ satisfies $i_{n}^{\prime}=i_{n}$ for all $n \in \mathbb{Z}$. In this way we define a map $\Xi_{\lambda_{t}, \lambda^{\prime}}: \hat{\Lambda}_{\lambda_{t}} \rightarrow \hat{\Lambda}_{\lambda^{\prime}}$ by $\Xi_{\lambda_{t}, \lambda^{\prime}}: \hat{x} \mapsto \hat{x}^{\prime}$.

It suffices to show that the point $\hat{x}^{\prime}=\left(x_{1}^{\prime}, x_{2}^{\prime}, x_{3}^{\prime}\right)$ defined by

$$
x_{1}^{\prime}=\frac{1-\lambda^{\prime}}{\lambda^{\prime}} \sum_{n=1}^{\infty} i_{-n}\left(\lambda^{\prime}\right)^{n}, \quad x_{2}^{\prime}=\frac{\gamma-1}{\gamma} \sum_{n=0}^{\infty} i_{n} \gamma^{n}, \quad x_{3}^{\prime}=\frac{1-\tau}{\tau} \sum_{n=1}^{\infty} i_{-n} \tau^{n}
$$

satisfies $\hat{f}_{\lambda^{\prime}}^{n}\left(\hat{x}^{\prime}\right) \in \hat{Q}_{i_{n}}$ for all $n \in \mathbb{Z}$. Then this implies that $\hat{x}^{\prime} \in \hat{\Lambda}_{\lambda^{\prime}}$. A change of $\lambda$ has only influence on the second coordinate of $\hat{f}(\hat{x})$. Since the local stable manifolds are parallel and oriented in the direction of the second coordinate it suffices to check that when changing $\lambda$, the second coordinate never move over the vertical discontinuity $S_{\mathrm{v}}$. We do this by an estimate of the derivative $\frac{\mathrm{d}}{\mathrm{d} \lambda}\left(\frac{1-\lambda}{\lambda} \sum_{n=0}^{\infty} i_{-n} \lambda^{n}\right)$. A simple calculation gives that

$$
\begin{aligned}
\left|\frac{\mathrm{d}}{\mathrm{d} \lambda}\left(\frac{1-\lambda}{\lambda} \sum_{n=0}^{\infty} i_{-n} \lambda^{n}\right)\right| & \leq\left|\frac{1}{\lambda^{2}} \sum_{n=0}^{\infty} i_{n} \lambda^{n}\right|+\left|\frac{1-\lambda}{\lambda} \sum_{n=1}^{\infty} n i_{n} \lambda^{n-1}\right| \\
& \leq \frac{1}{\lambda^{2}} \sum_{n=0}^{\infty} \lambda^{n}+\frac{1-\lambda}{\lambda} \sum_{n=1}^{\infty} n \lambda^{n-1}=\frac{1+\lambda}{\lambda^{2}(1-\lambda)}<15,
\end{aligned}
$$

if $\frac{1}{2}<\lambda<\frac{3}{4}$. This implies that

$$
\left|x_{1}-x_{1}^{\prime}\right| \leq \sup _{\lambda}\left|\frac{\mathrm{d}}{\mathrm{d} \lambda}\left(\frac{1-\lambda}{\lambda} \sum_{n=0}^{\infty} i_{-n} \lambda^{n}\right)\right|\left|\lambda_{t}-\lambda^{\prime}\right|<15\left|I_{t}\right| \leq \alpha .
$$


This means that $\hat{x}^{\prime}$ does not cross the vertical piece of the singularity and hence stays on the same side of the singularity as $\hat{x}$. Similarly one shows that any iterate $\hat{f}_{\lambda^{\prime}}^{n}\left(\hat{x}^{\prime}\right)$ of stays on the same side of the singularity as $\hat{f}_{\lambda_{t}}^{n}(\hat{x})$.

Remark. The partition of I into subintervals is arbitrary so in fact the symbolic spaces coincide for any $\lambda, \lambda^{\prime} \in I$.

We have shown that the symbolic space $\Sigma_{\lambda, \gamma, \kappa}$ does not change when $\lambda$ varies. We also need to estimate how the measure $\hat{\mu}_{\mathrm{SBR}}^{\lambda}$ changes.

Lemma 3. There is a constant $c_{1}>0$ such that for any $t$ and any $\lambda, \lambda^{\prime} \in I_{t}$

$$
c_{1}^{-1} \hat{\mu}_{\mathrm{SBR}}^{\lambda_{t}}\left(C_{\lambda_{t}}\right) \leq \hat{\mu}_{\mathrm{SBR}}^{\lambda}\left(C_{\lambda}\right) \leq c_{1} \hat{\mu}_{\mathrm{SBR}}^{\lambda_{t}}\left(C_{\lambda_{t}}\right) \text {, }
$$

for any cylinder set of the form $C_{\lambda}={ }_{k}\left[i_{k} i_{k+1} \cdots i_{n}\right]_{n}=\bigcap_{j=k}^{n} \hat{f}_{\lambda}^{-j}\left(\hat{Q}_{i_{j}}\right), k<n$.

Proof. Since there are only finitely many inverse images of the set $S_{\mathrm{v}}$, all other inverse images of the singularity will consist of a horizontal line. As $\lambda$ varies over $I$ these horizontal lines are not changed, only the inverse images of $S_{\mathrm{v}}$ changes. There is thus a constant $c_{1}$, independent of $t$, such that for any $\lambda \in I_{t}$ and any cylinder set of the form $C_{\lambda}={ }_{k}\left[i_{k} i_{k+1} \cdots i_{n}\right]_{n}=\bigcap_{j=k}^{n} \hat{f}_{\lambda}^{-j}\left(\hat{Q}_{i_{j}}\right), 0 \leq k<n$ we have

$$
c_{1}^{-1} \hat{\nu}\left(C_{\lambda_{t}}\right) \leq \hat{\nu}\left(C_{\lambda}\right) \leq c_{1} \hat{\nu}\left(C_{\lambda_{t}}\right)
$$

Especially $c_{1}^{-1} \hat{\nu}\left(\hat{f}_{\lambda_{t}}^{-m}\left(C_{\lambda_{t}}\right)\right) \leq \hat{\nu}\left(\hat{f}_{\lambda}^{-m}\left(C_{\lambda}\right)\right) \leq c_{1} \hat{\nu}\left(\hat{f}_{\lambda_{t}}^{-m}\left(C_{\lambda_{t}}\right)\right)$ for any $m \in \mathbb{N}$ and so

$$
c_{1}^{-1} \hat{\mu}_{\mathrm{SBR}}^{\lambda_{t}}\left(C_{\lambda_{t}}\right) \leq \hat{\mu}_{\mathrm{SBR}}^{\lambda}\left(C_{\lambda}\right) \leq c_{1} \hat{\mu}_{\mathrm{SBR}}^{\lambda_{t}}\left(C_{\lambda_{t}}\right),
$$

for any cylinder set of the form $C_{\lambda}={ }_{k}\left[i_{k} i_{k+1} \cdots i_{n}\right]_{n}=\bigcap_{j=k}^{n} \hat{f}_{\lambda}^{-j}\left(\hat{Q}_{i_{j}}\right)$ with $k<n$.

Remark. Lemma 3 is also valid for the conditional measures on the stable manifold.

Since the entropy of the conditional measures are a.s. equal that of the measure, the Shannon-McMillan-Breiman theorem implies that given $\varepsilon>0$, there exists a number $n_{0}(\lambda, \hat{x}, \varepsilon)$ such that

$$
\hat{\mu}_{\mathrm{SBR}}^{\lambda, \mathrm{s}, \hat{x}}\left(\left\{\hat{y} \mid \hat{\mu}_{\mathrm{SBR}}^{\lambda, \mathrm{s}, \hat{x}}\left(\hat{R}_{\lambda}^{q}(\hat{y})\right)<e^{-q \log (\gamma-\varepsilon)}, q>n_{0}(\lambda, \hat{x}, \varepsilon)\right\}\right)>1-\varepsilon .
$$

Lusin's Theorem implies that there is a number $n_{1}(\varepsilon)$ and a set $\hat{\Omega}_{0, \lambda_{t}}$ such that $n_{1}(\varepsilon) \geq n_{0}\left(\lambda_{t}, \hat{x}, \varepsilon\right)$ when $\hat{x} \in \hat{\Omega}_{0, \lambda_{t}}$ and $\hat{\mu}_{\mathrm{SBR}}^{\lambda_{t}}\left(\hat{\Omega}_{0, \lambda_{t}}\right)>1-\varepsilon$. We put

$$
\begin{aligned}
\hat{\Omega}_{\lambda_{t}}=\left\{\hat{y} \in \hat{\Omega}_{0, \lambda_{t}} \cap \hat{W}^{\mathrm{S}}(\hat{x}) \mid \hat{x} \in \Omega_{0, \lambda_{t}}\right. \text { and } & \\
& \left.\hat{\mu}_{\mathrm{SBR}}^{\lambda, \mathrm{s}, \hat{x}}\left(\hat{R}_{\lambda}^{q}(\hat{y})\right)<e^{-q \log (\gamma-\varepsilon)}, q>n_{1}(\varepsilon)\right\} .
\end{aligned}
$$

Then $\hat{\mu}_{\mathrm{SBR}}^{\lambda_{t}}\left(\hat{\Omega}_{\lambda_{t}}\right)>1-2 \varepsilon$.

Put $\hat{\Omega}_{\lambda}=\Xi_{\lambda_{t}, \lambda}\left(\hat{\Omega}_{t}\right)$ for $\lambda \in I_{t}$. By Lemma 3 it follows that if $\hat{y} \in \hat{\Omega}_{\lambda}$ then

$$
\hat{\mu}_{\mathrm{SBR}}^{\lambda}\left(\hat{R}_{\lambda}^{q}(\hat{y})\right)<c_{1} e^{-q \log (\gamma-\varepsilon)}, q>n_{1}(\varepsilon)
$$


and

$$
\hat{\mu}_{\mathrm{SBR}}^{\lambda}\left(\hat{\Omega}_{\lambda}\right)>c_{1}^{-1} \hat{\mu}_{\mathrm{SBR}}^{\lambda_{t}}\left(\Omega_{\lambda_{t}}\right)>c_{1}^{-1}(1-2 \varepsilon) .
$$

Take a $\lambda_{0} \in I$ and $\hat{x}_{\lambda_{0}} \in \hat{\Omega}_{\lambda_{0}}$. Define $\hat{x}\left(\lambda_{0}\right)=\hat{x}_{\lambda_{0}}$ and for $\lambda \in I$ define $\hat{x}(\lambda)$ so that $\rho_{\lambda, \gamma, \kappa}^{-1}(\hat{x}(\lambda))=\rho_{\lambda_{0}, \gamma, \kappa}^{-1}\left(\hat{x}\left(\lambda_{0}\right)\right)$. Then $\hat{x}$ is continuous. By Lemma 1

$$
\begin{aligned}
& T_{\underline{i}, t, k}=\int_{I_{t}} \int_{\hat{\Omega}_{\lambda} \cap \hat{R}_{\underline{\underline{1}}}^{k}(\lambda)} \int_{\hat{\Omega}_{\lambda} \cap \hat{R}_{\underline{\underline{i}}-1}^{k}(\lambda)} \chi_{\left\{\left|y_{1}-z_{1}\right|<r\right\}} \mathrm{d} \hat{\mu}_{\mathrm{SBR}}^{\lambda, \mathrm{s}, \hat{x}(\lambda)}(\hat{z}) \mathrm{d} \hat{\mu}_{\mathrm{SBR}}^{\lambda, \mathrm{s}, \hat{x}(\lambda)}(\hat{y}) \mathrm{d} \lambda \\
& \leq c_{1}^{2} \int_{I_{t}} \int_{\hat{\Omega}_{\lambda_{t}} \cap \hat{R}_{\underline{1}}^{k}\left(\lambda_{t}\right)} \int_{\hat{\Omega}_{\lambda_{t}} \cap \hat{R}_{\underline{i}-1}^{k}\left(\lambda_{t}\right)} \chi_{\left\{\left|\Xi_{\lambda_{t}, \lambda}(\hat{y})_{1}-\Xi_{\lambda_{t}, \lambda}(\hat{z})_{1}\right|<r\right\}} \\
& \mathrm{d} \hat{\mu}_{\mathrm{SBR}}^{\lambda_{t}, \mathrm{~s}, \hat{x}\left(\lambda_{t}\right)}(\hat{z}) \mathrm{d} \hat{\mu}_{\mathrm{SBR}}^{\lambda_{t}, \mathrm{~s}, \hat{x}\left(\lambda_{t}\right)}(\hat{y}) \mathrm{d} \lambda \\
& =c_{1}^{2} \int_{\hat{\Omega}_{\lambda_{t}} \cap \hat{R}_{\underline{1}}^{k}\left(\lambda_{t}\right)} \int_{\hat{\Omega}_{\lambda_{t}} \cap \hat{R}_{\underline{i}-1}^{k}\left(\lambda_{t}\right)} \int_{I_{p}} \chi_{\left\{\left|\Xi_{\lambda_{t}, \lambda}(\hat{y})_{1}-\Xi_{\lambda_{t}, \lambda}(\hat{z})_{1}\right|<r\right\}} \\
& \mathrm{d} \lambda \mathrm{d} \hat{\mu}_{\mathrm{SBR}}^{\lambda_{t}, \mathrm{~s}, \hat{x}\left(\lambda_{t}\right)}(\hat{z}) \mathrm{d} \hat{\mu}_{\mathrm{SBR}}^{\lambda_{t}, \mathrm{~s}, \hat{x}\left(\lambda_{t}\right)}(\hat{y}) \\
& \leq c_{2}\left(\gamma^{-1}+\varepsilon\right)^{-k} r \hat{\mu}_{\mathrm{SBR}}^{\lambda_{t}, \mathrm{~s}, \hat{x}\left(\lambda_{t}\right)}\left(\hat{R}_{\underline{i 1}}^{k}\left(\lambda_{t}\right)\right) \hat{\mu}_{\mathrm{SBR}}^{\lambda_{t}, \mathrm{~s}, \hat{x}\left(\lambda_{t}\right)}\left(\hat{R}_{\underline{i}-1}^{k}\left(\lambda_{t}\right)\right) \\
& \leq c_{2} r\left(\gamma^{-1}+\varepsilon\right)^{-k}(\gamma-\varepsilon)^{-k} \hat{\mu}_{\mathrm{SBR}}^{\lambda_{t}, \mathrm{~S}, \hat{x}\left(\lambda_{t}\right)}\left(\hat{R}_{\underline{i 1}}^{k}\left(\lambda_{t}\right)\right) \text {. }
\end{aligned}
$$

Since

$$
\hat{\Lambda}_{\lambda} \times \hat{\Lambda}_{\lambda}=\bigcup_{k=0}^{\infty} \bigcup_{\underline{i}} \hat{R}_{\underline{i} 1}^{k}(\lambda) \times \hat{R}_{\underline{i}-1}^{k}(\lambda)
$$

we can proceed as follows

$$
\begin{aligned}
\int_{I} \int_{\hat{\Omega}_{\lambda}} \int_{\hat{\Omega}_{\lambda}} \chi_{\left\{\left|y_{1}-z_{1}\right|<r\right\}} \mathrm{d} \hat{\mu}_{\mathrm{SBR}}^{\lambda, \mathrm{s}, \hat{x}(\lambda)}(z) \mathrm{d} \hat{\mu}_{\mathrm{SBR}}^{\lambda, \mathrm{s}, \hat{x}(\lambda)}(y) \mathrm{d} \lambda \\
\quad=\sum_{t=1}^{p} \sum_{k=0}^{\infty} \sum_{\underline{i}} T_{\underline{i}, t, k} \\
\quad \leq \sum_{t=1}^{p} \sum_{k=0}^{\infty} \sum_{\underline{i}} c_{3} r\left(\gamma^{-1}+\varepsilon\right)^{-k}(\gamma-\varepsilon)^{-k} \hat{\mu}_{\mathrm{SBR}}^{\lambda_{t}, \mathrm{~s}, \hat{x}\left(\lambda_{t}\right)}\left(\hat{R}_{\underline{i} 1}^{k}\left(\lambda_{t}\right)\right) \\
\quad \leq \sum_{t=1}^{p} \sum_{k=0}^{\infty} c_{3} r\left(\gamma^{-1}+\varepsilon\right)^{-k}(\gamma-\varepsilon)^{-k} \leq \sum_{t=1}^{p} c_{4} r=c_{5} r .
\end{aligned}
$$

Hence

$$
\liminf _{r \rightarrow 0} \frac{1}{r} \int_{I} \int_{\hat{\Omega}_{\lambda}} \int_{\hat{\Omega}_{\lambda}} \chi_{\left\{\left|y_{1}-z_{1}\right|<r\right\}} \mathrm{d} \hat{\mu}_{\mathrm{SBR}}^{\lambda, \mathrm{s}, \hat{x}(\lambda)}(\hat{z}) \mathrm{d} \hat{\mu}_{\mathrm{SBR}}^{\lambda, \mathrm{s}, \hat{x}(\lambda)}(\hat{y}) \mathrm{d} \lambda \leq c_{5} .
$$

This is independent of the choice of $\hat{x}: I \rightarrow Q$ so this proves Theorem 1 .

\section{Decay of correlations}

Consider the following class of Hölder continuous functions defined on $Q$

$$
\mathcal{H}_{\eta}=\left\{\phi: Q \rightarrow \mathbb{R}|\exists C:| \phi(x)-\phi(y) \mid<C d(x, y)^{\eta} \forall x, y \in Q\right\} .
$$

We will show that $f$ has exponential decay of correlations for functions in $\mathcal{H}_{\eta}$. More precisely, we will show the following theorem. 
Theorem 2. Let $(\lambda, \gamma, \kappa) \in P$. There is a $\tau=\tau(\eta)<1$ such that if $\phi, \psi \in \mathcal{H}_{\eta}$ then there is a $C=C(\phi, \psi)$ such that

$$
\left|\int\left(\phi \circ f_{\lambda, \gamma, k}^{n}\right) \psi \mathrm{d} \mu_{\mathrm{SBR}}-\int \phi \mathrm{d} \mu_{\mathrm{SBR}} \int \psi \mathrm{d} \mu_{\mathrm{SBR}}\right|<C \tau^{n} .
$$

In [17], Young introduces a general scheme for proving exponential decay of correlations. The method is to find a set $\Theta$ with nice hyperbolic properties and a hyperbolic product structure;

$$
\Theta=\left(\bigcup_{\omega^{\mathrm{s}} \in \Gamma^{\mathrm{s}}} \omega^{\mathrm{s}}\right) \bigcap\left(\bigcup_{\omega^{\mathrm{u}} \in \Gamma^{\mathrm{u}}} \omega^{\mathrm{u}}\right)
$$

where $\Gamma^{\mathrm{s}}$ and $\Gamma^{\mathrm{u}}$ are collections of stable and unstable curves respectively, and a return time $R: \Theta \rightarrow \mathbb{N}$ such that $f^{R(\cdot)}(\cdot): \Theta \rightarrow \Theta$.

For $\omega \in \Gamma^{\mathrm{u}}$ let $\nu_{\omega}$ denote the conditional measure of the Lebesgue measure $\nu$ with respect to the curve $\omega$. If the conditions

$$
\begin{aligned}
& \nu_{\omega}(\omega \cap \Theta)>0, \text { for each } \omega \in \Gamma^{\mathrm{u}} \\
& \nu\{x \in \Theta \mid R(x)>n\}<C \theta^{n}, \text { for some } C \text { and } \theta<1, \\
& \left(f^{n}, Q\right) \text { is ergodic for each } n>0,
\end{aligned}
$$

and some other regularity conditions are satisfied then this is sufficient to conclude exponential decay of correlations for Hölder continuous functions.

Among other examples in [17], Young shows how to find the set $\Theta$ and the return time $R$ for a class of piecewise $C^{2}$ hyperbolic maps in two dimensions. This class of maps is different from our class but still the method can be used to construct $\Theta$ and $R$. We give the construction of $\Theta$ and $R$ below.

The fact that $(\lambda, \gamma, \kappa) \in P$ makes the construction of $\Theta$ easier. This is because there is a uniform estimate of the length of the local stable manifolds for these parameters. From Section 4 we conclude that $W_{\alpha}^{\mathrm{s}}(x)$ exists for all $x \in \Lambda$. Theorem 2 is true also if $(\lambda, \gamma, \kappa) \notin P$ but then we have to work with the set

$$
D_{\delta}^{+}=\left\{x \mid d\left(f^{n}(x), S\right)>\delta \gamma^{-n} \forall n \geq 0\right\},
$$

which would have made the construction of $\Theta$ somewhat more technical.

We will now proceed with the construction of $\Theta$ and $R$. It is important to gather enough expansion in the unstable direction. For this purpose we take an $N \in \mathbb{N}$ such that $\gamma^{N}>2 e^{\frac{1}{e}}$.

Choose $\delta>0$ so that for any curve $\omega$ in the unstable direction with length not greater than $\delta$ the set $f^{N}(\omega)$ consists of at most two connected components. This can be done since we can choose $\delta$ to be the smallest distance between the lines in the set $\cup_{n=0}^{N} f^{-n}\left(S_{\mathrm{h}}\right)$.

Take $0<\delta_{0}<\frac{1}{6} \delta$ to be so small that the set

$$
A_{\delta_{0}}=\left\{x \in \Theta \mid W_{2 \delta_{0}}^{\mathrm{u}}(x) \text { exists }\right\}
$$

has positive Lebesgue measure. For any $x \in A_{\delta_{0}}$ we define $\Omega(x)=W_{\delta_{0}}^{\mathrm{u}}(x)$. Put

$$
\begin{aligned}
\Gamma^{\mathrm{s}}(x) & =\left\{W^{\mathrm{s}}(y) \mid y \in \Omega(x)\right\}, \\
\Gamma^{\mathrm{u}}(x) & =\left\{W^{\mathrm{u}}(z) \mid z \in A_{\delta_{0}}, W^{\mathrm{u}}(z) \cap W^{\mathrm{s}} \neq \emptyset, \forall W^{\mathrm{s}} \in \Gamma^{\mathrm{s}}(x)\right\} .
\end{aligned}
$$


We let $\Theta(x)$ be the hyperbolic set with the product structure defined by $\Gamma^{\mathrm{s}}(x)$ and $\Gamma^{\mathrm{u}}(x)$.

For any $x \in A_{\delta_{0}}$ we let $Q(x)$ be the smallest open rectangle containing $\Theta(x)$. The open sets $\{Q(x)\}_{x \in A_{\delta_{0}}}$ form an open covering of $A_{\delta_{0}}$. Since $A_{\delta_{0}}$ is compact we can select a finite subcover $\left\{Q\left(x_{i}\right)\right\}_{i=1}^{r}$. The sets $\left\{\Theta\left(x_{i}\right)\right\}_{i=1}^{r}$ will then cover $A_{\delta_{0}}$. We will write $\Theta_{i}$ for $\Theta\left(x_{i}\right)$.

We define the return time $R$ to $\cup \Theta_{i}$ on a subset of the sets $\Theta_{i}$. Let $i$ be fixed. We iterate the map $f^{N}$ and consider the connected components of the set $\left(f^{N}\right)^{n}\left(\Omega\left(x_{i}\right)\right)$.

We construct for each $n \in \mathbb{N}$ a partition $\mathcal{P}_{n}^{i}$ of $\Omega\left(x_{i}\right) \backslash\{R \leq n\}$ into connected curves with the property that if $\omega \in \mathcal{P}_{n}^{i}$ then $\left(f^{N}\right)^{n}(\omega)$ is a connected curve of length $<6 \delta_{0}$.

Let $\mathcal{P}_{0}^{i}$ be the trivial partition, $\mathcal{P}_{0}^{i}=\left\{\Omega\left(x_{i}\right)\right\}$. Assume that $\mathcal{P}_{n-1}^{i}$ is defined. Let $\omega \in \mathcal{P}_{n-1}^{i}$. Since $\left(f^{N}\right)^{n-1}(\omega)$ is a connected curve of length $<6 \delta_{0}$ the set $\left(f^{N}\right)^{n}(\omega)$ consists of at most two connected components. Let $\left\{\omega_{j}^{\prime}\right\}_{j=1,2}$ be the corresponding components of $\omega$. If $\left(f^{N}\right)^{n}\left(\omega_{j}\right)$ has length $<6 \delta_{0}$ then we put $\omega_{j}$ in $\mathcal{P}_{n}^{i}$. If however $\left(f^{N}\right)^{n}\left(\omega_{j}\right)$ has length $\geq 6 \delta_{0}$ then there is a $k$ such that $\left(f^{N}\right)^{n}\left(\omega_{j}\right)$ crosses $Q\left(x_{k}\right)$ with segments of length $\geq \delta_{0}$ sticking out on each side of $Q\left(x_{k}\right)$. Since $\left|\left(f^{N}\right)^{n}\left(\omega_{j}\right)\right|>6 \delta_{0}$ we have $\left(f^{N}\right)^{n}\left(\omega_{j}\right) \in \Gamma^{\mathrm{u}}\left(x_{k}\right)$ and this implies that $\left(f^{N}\right)^{n}\left(\omega_{j}\right) \cap Q\left(x_{k}\right) \subset \Theta_{k}$. We define $R=n$ on $\omega_{j} \cap\left(f^{N}\right)^{-n}\left(\Theta_{k}\right)$. In this way we get two pieces of length $>\delta_{0}$ on each side of $\left(f^{N}\right)^{n}\left(\omega_{j} \backslash\{R=n\}\right)$. We partition $\omega \backslash\{R=n\}$ into $\left\{\omega_{j, k}\right\}_{k=1}^{m}$ such that $\delta_{0}<\left(f^{N}\right)^{n}\left(\omega_{j, k}\right)<6 \delta_{0}$ and put $\left\{\omega_{j, k}\right\}$ in $\mathcal{P}_{n}^{i}$.

Finally, if $\omega \in \mathcal{P}_{n-1}$ then we define $R=n$ on the set

$$
S_{\omega}=\left(\bigcup_{\omega^{\mathrm{s}} \in \Gamma^{\mathrm{s}}(\omega)} \omega^{\mathrm{s}}\right) \bigcap\left(\bigcup_{\omega^{\mathrm{u}} \in \Gamma^{\mathrm{u}}} \omega^{\mathrm{u}}\right) \subset \Theta_{i},
$$

where $\Gamma^{\mathrm{s}}(\omega)$ is the set of local stable manifolds in $\Gamma^{\mathrm{s}}\left(x_{i}\right)$ which has non-empty intersection with $\omega$. The uniform estimate on the length of $\omega_{s} \in \Gamma^{\mathrm{s}}$ implies that $\left(f^{N}\right)^{n}\left(S_{\omega}\right) \subset \Theta_{k}$.

Lemma 4. Let $\Omega=\Omega\left(x_{i}\right)$ for some $x_{i}$. There exists $C>0$ and $\theta_{1}<1$ such that

$$
\nu_{\Omega}\{R>n\} \leq C \theta_{1}^{n} .
$$

Proof. Let $T_{1}(x)$ be the smallest $n \geq 1$ such that if $\omega \in \mathcal{P}_{n-1}$ is the component containing $x$ then $\left|\left(f^{N}\right)^{n}(\omega)\right| \geq 6 \delta_{0}$. If there is no such $n$ then we say that $T_{1}(x)$ is not defined. Note that $T_{1} \leq R$

Suppose that $T_{k}(x)$ has been defined. Then we define $T_{k+1}(x)$ to be the smallest $n>T_{k}(x)$ such that if $\omega \in \mathcal{P}_{n-1}$ is the component containing $x$ then $\left|\left(f^{N}\right)^{n}(\omega)\right| \geq 6 \delta_{0}$. Let $\mathcal{T}_{k}=\left\{T_{k}\right.$ is defined $\}$.

Each time a segment is stretched to a length over $6 \delta_{0}$ a piece of length $2 \delta_{0}$ returns to one of the sets $\Theta_{i}$. This implies that if $\omega \in \mathcal{P}_{n-1}$ and $\left.T_{1}\right|_{\omega}=n$ then $\left|\left(f^{N}\right)^{n}(\omega)\right|<\gamma^{N} 6 \delta_{0}$ and so

$$
\frac{\nu_{\Omega}\left(\omega \cap\left\{R=T_{1}\right\}\right)}{\nu_{\Omega}(\omega)}>\frac{2 \delta_{0}}{6 \delta_{0} \gamma^{N}}=\frac{1}{3 \gamma^{N}} .
$$

Hence

$$
\frac{\nu_{\Omega}\left(\omega \cap\left\{R>T_{1}\right\}\right)}{\nu_{\Omega}(\omega)}<1-\frac{1}{3 \gamma^{N}}=\theta_{2} .
$$


This means that $\frac{\nu_{\Omega}\left(\mathcal{T}_{2}\right)}{\nu_{\Omega}\left(\mathcal{T}_{1}\right)}<\theta_{2}$. Similarly we get

$$
\frac{\nu_{\Omega}\left(\mathcal{T}_{k+1}\right)}{\nu_{\Omega}\left(\mathcal{T}_{k}\right)}<\theta_{2}
$$

This implies that $\nu_{\Omega}\left(\mathcal{T}_{k}\right)<\theta_{2}^{k}$ and $\nu_{\Omega}\left\{R>T_{k}\right\}<\theta_{2}^{k}$.

For any $k$

$$
\{R>n\} \subset\left\{T_{k} \geq n\right\} \cup\left\{T_{k}<n<R\right\} .
$$

There is a number $M$ such that if $\omega \in \mathcal{P}_{n-1}$ then $\omega \backslash\{R=n\}$ can be covered by less than $M$ elements from $\mathcal{P}_{n}$.

Let $K_{p}=\left\{k_{i}\right\}_{i=1}^{p}$ where $k_{1}<k_{2}<\cdots<k_{p}$ with $k_{p} \geq n$ and $k_{p-1}<n$. Consider the set $A_{K_{p}}=\left\{T_{i}=k_{i}, i=1,2, \ldots, p\right\}$. It can be covered by less than $2^{k_{p}} M^{p}$ elements from $\mathcal{P}_{k_{p}}$. Since the length of $\Omega$ is $2 \delta_{0}$ we have

$$
\nu_{\Omega}\left(A_{K_{p}}\right) \leq \frac{2^{k_{p}} M^{p} 6 \delta_{0}\left(\gamma^{N}\right)^{-k_{p}}}{2 \delta_{0}}=3 M^{p}\left(\frac{2}{\gamma^{N}}\right)^{k_{p}} .
$$

If $p \ll n$ then by Stirling's formula

$$
\left(\begin{array}{l}
n \\
p
\end{array}\right) \approx \frac{n^{n+\frac{1}{2}} e^{-n}}{p !(n-p)^{n-p+\frac{1}{2}} e^{-n+p}}=\frac{e^{-p} n^{p}}{p !}\left(1-\frac{p}{n}\right)^{p-n}
$$

and so if $\varepsilon$ is small enough

$$
\begin{aligned}
\sum_{p=0}^{[\varepsilon n]}\left(\begin{array}{l}
n \\
p
\end{array}\right) & \leq C_{1} \sum_{p=0}^{[\varepsilon n]} \frac{e^{-p} n^{p}}{p !}\left(1-\frac{p}{n}\right)^{p-n} \\
& <C_{1}(1-\varepsilon)^{-n} \sum_{p=0}^{[\varepsilon n]} \frac{\left(\frac{n}{e}(1-\varepsilon)\right)^{p}}{p !}<C_{1}(1-\varepsilon)^{-n} e^{\frac{n}{e}(1-\varepsilon)} .
\end{aligned}
$$

Choose $\varepsilon$ so small that $\theta_{3}=\frac{e^{\frac{1-\varepsilon}{e}} M^{\varepsilon} 2}{(1-\varepsilon) \gamma^{N}}<1$. This can be done because of the choice of $N$. Then

$$
\begin{aligned}
\nu_{\Omega}\left\{T_{[\varepsilon n]}>n\right\} & \leq \sum_{p=0}^{[\varepsilon n]} \sum_{K_{p}} \nu_{\Omega}\left(A_{K_{p}}\right) \leq \sum_{p=1}^{[\varepsilon n]}\left(\begin{array}{l}
n \\
p
\end{array}\right) 3 M^{p} \sum_{k_{p}=n}^{\infty}\left(\frac{2}{\gamma^{N}}\right)^{k_{p}} \\
& \leq C_{2}\left(\frac{e^{\frac{1-\varepsilon}{e}} M^{\varepsilon} 2}{(1-\varepsilon) \gamma^{N}}\right)^{n}=C_{2} \theta_{3}^{n} .
\end{aligned}
$$

We use (7) to approximate $\nu_{\Omega}\{R>n\}$. We choose $k=\varepsilon n$ and get

$$
\nu_{\Omega}\{R>n\} \leq C_{2} \theta_{3}^{n}+\theta_{2}^{\varepsilon n} \leq C \theta_{1}^{n} .
$$

We have proved that the return time $R$ decays exponentially. This is not quite what we want. $R$ is the time needed for a piece of $\Theta_{i}$ to return to some $\Theta_{j}$, but we would need $R$ to be the return time from $\Theta_{i}$ to $\Theta_{i}$. Arguing as in [17], we can choose $\Theta_{*}=\Theta_{i}$ for some $i$ such that the return time $R_{*}$ of $\Theta_{*}$ satisfies $\nu_{\omega}\{R>n\}<C \theta^{n}$ for some $\theta<1$. This is sufficient to conclude Theorem 2 . Note that the SBR-measure constructed with the method in [17] is the same measure as the SBR-measure constructed in Section 2. 


\section{References}

[1] J. C. Alexander, J. A. Yorke, Fat baker's transformations, Ergod. Th. \& Dynam. Sys. 4 (1984), 1-23

[2] J. Buzzi, Absolutely continuous invariant measures for generic multidimensional piecewise affine expanding maps, Internat. J. Bifur. Chaos Appl. Sci. Engrg. 9 (1999), 1743-1750

[3] J. Buzzi, Absolutely continuous invariant probability measures for arbitrary expanding piecewise $\mathbb{R}$-analytic mappings of the plane, Ergod. Th. \& Dynam. Sys. 20 (2000), 697-708

[4] J. Buzzi, G Keller, Zeta functions and transfer operators for multidimensional piecewise affine and expanding maps, Ergodic Theory Dynam. Systems 21 (2001), 689-716

[5] V. P. Belykh, Models of discrete systems of phase synchronization, Systems of Phase Synchronization. Eds. V. V. Shakhildyan and L. N. Belynshina. Radio i Svyaz, Moscow, 1982, 61-176

[6] P. Erdös, On a family of symmetric Bernoulli convolutions, Amer. J. Math. 61 (1939), 974-976

[7] J. Neunhäuserer, Dimension theoretical properties of generalized Baker's transformations, Nonlinearity 15, 1299-1307, 2002

[8] Y. Peres, B. Solomyak, Absolute continuity of Bernoulli convolutions, a simple proof, Math. Research Letters 3 (1996), 231-236.

[9] Ya. B. Pesin, Dynamical systems with generalized hyperbolic attractors: hyperbolic, ergodic and topological properties, Ergod. Th. \& Dynam. Sys. 12 (1992), 123-151

[10] E. A. Sataev, Invariant measures for hyperbolic maps with singularities, Russian Math. Surveys 47 (1992), 191-251

[11] E. A. Sataev, Ergodic properties of the Belykh map, J. Math. Sci. (New York) 95 (1999), 2564-2575

[12] J. Schmeling, A dimension formula for endomorphisms - the Belykh family, Ergod. Th. \& Dynam. Sys. 18 (1998), 1283-1309

[13] J. Schmeling, S. Troubetzkoy, Dimension and invertibility of hyperbolic endomorphisms with singularities, Ergod. Th. \& Dynam. Sys. 18 (1998), $1257-1282$

[14] B. Solomyak, On the random series $\sum \pm \lambda^{i}$ (an Erdös problem), Annals of Math. 142 (1995), 611-625.

[15] M. Tsujii, Absolutely continuous invariant measures for piecewise realanalytic expanding maps on the plane, Comm. Math. Phys. 208 (2000), $605-622$

[16] M. Tsujii, Absolutely continuous invariant measures for expanding piecewise linear maps, Invent. Math. 143 (2001), 349-373

[17] L.-S. Young, Statistical properties of dynamical systems with some hyperbolicity, Annals of Math. 147 (1998), 585-650. 


Preprints in Mathematical Sciences 2005:29

ISSN 1403-9338

LUTFMA-5065-2005

Mathematics

Centre for Mathematical Sciences

Lund University

Box 118, SE-221 00 Lund, Sweden

http://www.maths.lth.se/ 